\title{
Legal Regulation of the Use of Digital Technologies in The Agricultural Economy
}

\author{
Kheda Mutazova ${ }^{1, *}$, Zhanati Salgirieva ${ }^{2}$ \\ ${ }^{1}$ Chechen State University named after A.A.Kadyrov, Grozny, Russia \\ ${ }^{2}$ Grozny State Oil Technical University named after Academician M.D. Millionshchikova, Grozny, Russian \\ Federation \\ *Corresponding author. Email: fu.ggni@mail.ru
}

\begin{abstract}
Digital technologies, including the Internet, mobile technologies and devices, data analytics, artificial intelligence, digital services and applications, are changing agriculture and the food system. And also must have a solid legal basis. Examples abound at different stages of the agri-food value chain: agricultural machinery automation allows for precise control of costs and reduces the demand for manual labor, remote satellite data and on-site sensors improve the accuracy and reduce the cost of monitoring crop growth and land or water quality, and traceability technologies and digital logistics services optimize agri-food supply chains.
\end{abstract}

Keywords: digital technologies, agriculture, food system, agricultural machinery automation, land or water quality, traceability technologies, digital logistics services.

\section{INTRODUCTION}

The adoption of digital technologies in agriculture is growing at a rapid pace. In fact, digital adoption across all industries is on the rise. The reason this is such a pervasive trend is because digital technology brings tremendous benefits to companies and individuals. In the agricultural industry, there are many digital technologies that fall under the category of "precision farming" [1]. Precision farming technologies are changing the way farmers grow crops and are increasingly being applied. Agricultural utilization is a data-driven methodology for managing and optimizing crop production. In recent years, agricultural producers have been introducing precision farming technologies for several reasons. A recent study from the University of Nebraska at Lincoln on the adoption of precision farming technology reflects this trend.

This study surveyed agricultural producers at various Nebraska Extension events on the digitalization of agriculture. The most widespread are responses from soil samples $(98 \%)$ and a computer with Internet access (94\%). Crop monitors and maps, as well as GPS navigation systems - over $80 \%$. Satellite and aerial photographs are becoming more and more popular among farmers [7-8].
In general, the introduction of precision farming technologies is quite large. Some of these technologies, such as the Internet or tablets, are not necessarily exclusively used in precision farming, but they are foundational technologies that allow for further adoption in the agro-industrial complex.

Consideration of the system "man - relief" is determined by the need to identify the role of the geographic environment of the location of the city on its growth and development and, in particular, consideration of the relief as the basis of the ecosystem "City" [1]. This position in science is new. Man created a city, his ecosystem, in accordance with his needs, in which he, a man, is the first systemforming link, and the other is the natural (geographical) environment. Their interaction forms an urban area and a specific natural and anthropogenic environment - an urban environment, or an urban planning system, in which the relief plays the role of a structural planning framework and is the basis of an ecological framework [2].

\section{RESEARCH METHODOLOGY}

In the era of digitalization and communication technologies (ICT), such as mobile phones and computer technologies have revolutionized and a new paradigm for doing business and the use of services 
has emerged. However, there is still a significant digital divide within and between different countries [2-3].

All over the world, the number of mobile cellular subscribers has been growing lately. Yet 3.8 billion people are still offline. They are mainly located in rural and remote areas. One problem is that network coverage in rural areas remains limited. Despite the fact that $4 \mathrm{G}$ has become the most widespread mobile connection worldwide and $90 \%$ of the possibility of Internet access is through a $3 \mathrm{G}$ network or better, only about a third of the rural population is covered by $3 \mathrm{G}$ networks. Smartphones have become the main way for consumers to access the Internet. Using digital technology requires basic literacy, numeracy, and specialized technical knowledge and skills. People without such competencies may find themselves isolated from the information society, which is increasingly focused on digital technologies [5].

In rural areas, infrastructure and resources are often lacking, and the quality of education is also limited. This leads to a less efficient learning process. In addition, in many rural areas, young people are often required to work, leaving little time for study. Thus, the level of education is often low. Lack of basic literacy and numeracy is a major obstacle to the use of digital technology. In many countries, state policy is aimed at digitalizing the economy. This creates a favorable environment for the competition of the digital market and electronic services [4]. There is also a trend towards governments themselves, which are currently focusing on digitalization - "egovernment" - especially in health, education, the environment and employment. However, in order to create so-called digital government and governance, the program requires a high level of administrative capacity. Developing countries are often the ones with the least control over the process. Compared to just a decade ago, governments have made significant progress in expanding access to digital networks.

Now that we see this technology is widespread and will continue to be used by farmers, we can explore some of its benefits. The main value that crop technology brings include [5] t:

- efficient use of resources such as chemicals, fertilizers, water, fuel;

- increasing the quantity and quality of products;

- higher productivity on the same amount of land;

- reducing the impact on the environment;

-reduction of risks.

These benefits add tremendous value to the manufacturing process due to the increased yield that is a direct result of the integration of this technology.
Value added can be determined by analyzing projected yield increases, and in the same Goldman Sachs report, they calculated the dollar value added based on each of the major forms of cropping technology. The results are presented below.

Precision Fertilizer - \$65 Billion TAM (Total Target Market) with \$ 200 Billion value added based on $18 \%$ yield increase [5-6].

Precision Planting is a $\$ 45$ billion TAM with $\$$ 145 billion in value added with a $13 \%$ increase in yields.

Reducing compaction with smaller tractors - \$45 billion TAM with an added value of $\$ 145$ billion with a $13 \%$ increase in yield [7].

Precision Irrigation - \$ 35 Billion TAM with \$ 115 Billion added value on $10 \%$ yield increase.

Field monitoring, data management, etc. - \$35 billion TAM with a potential added value of $\$ 125$ billion [8].

Precision Spray - \$15 Billion TAM with \$50 Billion value added at $4 \%$ yield increase.

Digital technology can also help governments improve the efficiency and effectiveness of existing policies and programs, and design better ones. For example, free high-quality satellite imagery significantly reduces the cost of monitoring many agricultural operations. This could allow governments to move towards more targeted policies that pay (or punish) farmers based on observable environmental outcomes. In addition to monitoring compliance with environmental policies, digital technologies enable the automation of administrative processes in agriculture and the development of advanced public services, for example, in relation to extension services or advisory services.

Finally, digital technologies can support agricultural and food trade by connecting private sector suppliers to new markets and opening up new ways for governments to monitor and enforce standards, and provide faster and more efficient border procedures that are required for perishable goods. [9]

These technological advances can contribute to the achievement of more sustainable, productive and sustainable agricultural and food systems that better meet consumer needs. These benefits come both directly through the adoption of technology by sector actors (including service providers) and indirectly through the adoption of technology by governments for better policymaking. 


\section{RESULTS}

Three key questions highlight the actions needed by governments to realize the opportunities offered by digital technologies [6-7]:

First, how can government policies and programs adequately support digitalization in the agriculture and food sectors? Policymakers will need to consider the potential benefits, costs and risks, and understand the drivers of technology adoption so that interventions can target where market failure is occurring or the public interest.

How can governments use digital technology to design and implement more effective agricultural policies? This requires an understanding of how technology can help in various components of the policy cycle, and may require governments to expand their skills, invest in technology and training, or partner with other actors (both government and nongovernment).

How can digital technologies change the role of government? On the one hand, digital technologies can create new roles or responsibilities for governments, including for creating digital infrastructure (does the government have the ability to act as a provider or developer of rules for new digital infrastructure and under what circumstances); on the other hand, if technology can reduce information asymmetries and transaction costs, less government intervention may be required.

For policymakers, the challenge will be to shape policies and regulations in a way that facilitates digital opportunities. At the same time, and not just for the agricultural sector, digital technology raises questions about privacy, interoperability, and even potential liability issues that will need careful consideration.

Along with its many benefits, the agri-food value chain also faces some obstacles. The main problems are as follows:

- many new technologies are used or are beginning to be used in agriculture, but the level of implementation in Europe is still very low compared to other regions of the world, for example the USA [5].

- the basic requirements for the inclusion of most new technologies, broadband coverage and good internet connection, are unevenly distributed throughout Europe, especially in remote rural areas.

-small and medium-sized farmers strive for the profitability and reliability of new technologies. Resistance to change and the introduction of new technologies in agriculture may be related to the knowledge gap for agricultural needs. Regardless of age or sector, farmers seem to be equally sensitive to technology and its adoption. Their main goal is to benefit and reduce labor costs without disrupting their operations.

-small and medium-sized farmers have difficulty with investment opportunities.

Investing in new technologies is expensive and it is difficult for small and medium-sized enterprises to keep up with all technological trends. Therefore, to solve this problem, a methodology is being developed through public-private partnerships.

\section{CONCLUSIONS}

The farming technology revolution is driving an increase in the use of technology in the agricultural sector and a change in farming practices. In addition to more resource efficient agricultural production, we may notice that digital technologies, more specifically, technologies for increasing yields, are increasing. vertical integration in the food chain 71 . On the one hand, vertical integration optimizes the cost, efficiency and complementarity of resource suppliers (seed, fertilizer suppliers), while on the other hand, vertical integration tends to favor large food suppliers investing in agricultural technology.

Legal regulation of measures ensuring digital transformation, including agriculture, should be supported by updated legislative provisions of a general nature. So, for example, the Civil Code of the Russian Federation was supplemented by Article 783.1, which fixes the characteristic features of an agreement on the provision of information services. Article 160 of the Civil Code of the Russian Federation states that the written form of a transaction is also considered to be complied with if a person concludes a transaction using electronic or other technical means that make it possible to reproduce the contents of the transaction on a material medium unchanged, while the requirement for a signature is considered fulfilled if any a way to reliably identify the person who has expressed the will. Article 309 of the Civil Code of the Russian Federation is supplemented by part two, which establishes that: "the terms of the transaction may provide for the parties to fulfill the obligations arising from it upon the occurrence of certain circumstances ... by using information technologies determined by the terms of the transaction" [8]. The updated federal law "On Electronic Signatures" modified the system of certification centers; defined the concept and developed a mechanism for using a trusted time stamp; the possibility of using a "cloud" electronic signature is provided; introduced the legal framework of a trusted third party for internal and cross-border interaction. 


\section{REFERENCES}

[1] O. F. Balatsky, L. G. Melnik, A. F. Yakovlev, Economics and Quality of the Environment, p. 152 (2019).

[2] V. V. Bardyuk., L. S. Stoiko, On the Issue of Integrated Assessment of Surface Water Quality, p. 44 (2020).

[3] R. A. Gakaev, Mountain meadow landscapes of the Chechen Republic and features of their distribution, pp. 149-151 (2019).

[4] G. A. Bogdanovsky, Chemical Ecology, p. 421 (2019).

[5] S. D. Belyaev, Using water quality targets in water management planning, pp. 149-151 (2019).

[6] A. M. Vladimirov, F. A. Imanov, Principles for assessing the ecological flow of rivers.

[7] Digital Regulation Handbook: Geneva: International Telecommunication Union and the World Bank (2020).

[8] ITU and FAO. Status of Digital Agriculture in 18 countries of Europe and Central Asia. Geneva, Switzerland (2020).

[9] I. P. Chupina, The competitiveness of products as the object of a targeted strategic development of an economic entity, p. 142 (2020).

[10] P. B. Akmarov, Problematic issues of development of information technologies in Russia, pp. 177-180 (2019). 